\title{
The Ratio of Hmox1/Nrf2 mRNA Level in the Tumor Tissue Is a Predictor of Distant Metastasis in Colorectal Cancer
}

\author{
Liang-Che Chang, ${ }^{1}$ Chung-Wei Fan, ${ }^{2}$ Wen-Ko Tseng, ${ }^{2}$ Hui-Ping Chein, ${ }^{1}$ Tsan-Yu Hsieh, ${ }^{1}$ \\ Jim-Ray Chen, ${ }^{1}$ Cheng-Cheng Hwang, ${ }^{1}$ and Chung-Ching $\mathrm{Hua}^{3}$
}

${ }^{1}$ Department of Pathology, Chang Gung Memorial Hospital, Keelung, Taiwan

${ }^{2}$ Division of Colon and Rectal Surgery, Chang Gung Memorial Hospital, Keelung, Taiwan

${ }^{3}$ Department of Internal Medicine, Chang Gung Memorial Hospital, Keelung, Taiwan

Correspondence should be addressed to Chung-Ching Hua; hc2008@adm.cgmh.org.tw

Received 28 June 2016; Revised 30 September 2016; Accepted 1 November 2016

Academic Editor: Silvia Angeletti

Copyright (C) 2016 Liang-Che Chang et al. This is an open access article distributed under the Creative Commons Attribution License, which permits unrestricted use, distribution, and reproduction in any medium, provided the original work is properly cited.

\begin{abstract}
Heme oxygenase 1 (Hmoxl) plays an important role in the growth and spread of tumor, and its expression is regulated positively by Nrf2 [nuclear factor (erythroid-derived 2)-like 2; NFE2L2] and negatively by kelch-like ECH-associated protein 1 (Keap1) and by BTB and CNC homology 1 (Bach1). Both Hmoxl and Nrf2 contribute to distant metastasis of cancer. The mRNA levels of Hmoxl, Nrf2, Keap1, and Bach1 in the tumor and normal tissues of 84 subjects with colorectal cancer (CRC) were determined by real-time polymerase chain reaction. The tumor had lower Hmoxl but higher Bachl mRNA levels than the normal tissue. The correlations of Hmoxl with components of the Nrf2 pathway were not significant in the tumor tissue of CRC subjects with distant metastasis. The ratio of Hmoxl/Nrf2 mRNA level (by percentage) in the tumor tissue was lower in the subjects with distant metastasis ( $97.4 \%$ (84.4-111.1\%)) than in those without (101.0\% (92.7-136.5\%)) and was a predictor for distant metastasis in CRC (odds ratio: 0.83; 95\% confidence interval: 0.68-0.97) along with serum carcinoembryonic antigen (1.0027, 1.006-1.064). The mRNA level of Hmoxl in the tumor tissue of CRC is not correlated with that of the Nrf2 pathway molecules, and its ratio to the Nrf2 level may be useful for suggesting distant metastasis in CRC.
\end{abstract}

\section{Introduction}

Oxidative stress is an essential factor in the pathogenesis of gastrointestinal mucosal disease, including cancers [1], and may contribute to neoplastic transformation in colorectal cancer (CRC) through direct epithelial damage and genetic/ epigenetic alterations [2]. Heme oxygenase 1 (Hmoxl) can be induced by oxidative stress and may play a role in tumor induction, growth, or spread [3].

Hmoxl is one of the main effectors in cell responses regulated by the Nrf2 [nuclear factor (erythroid-derived 2)like 2; NFE2L2] pathway [4], which is one of the major cellular defense mechanisms against oxidative stress [5]. Nrf2 is a "cap 'n' collar" (CNC) basic leucine zipper transcription factor associated with its negative regulator, kelch-like ECHassociated protein 1 (Keapl), in the cytoplasm of unstressed cells, but is released from it and translocated to the nucleus under oxidative stress [6]. Once in the nucleus, Nrf2 competes with BTB (broad complex-tramtrack-bric-a-brac) and CNC homology 1 (Bach1) for binding small Maf proteins to form a heterodimer serving as a transcriptional activator that recognizes the antioxidant response element (ARE) in the promoters of Nrf2 itself, Keap1, Bach1, and many phase II detoxifying enzymes like Hmoxl [7]. Conversely, nuclear Hmoxl can bind to Nrf2 and stabilize it from glycogen synthase kinase $3 \beta$ - (GSK3 $\beta$-) mediated degradation [8]. Carbon monoxide induced by active Hmoxl can activate Nrf2 [9]. The molecules of the Nrf2 pathway interact with Hmoxl on the activity of promoter with ARE.

Hmoxl expression is mainly regulated by Nrf2. Hmoxl may counteract reactive oxygen species- (ROS-) mediated carcinogenesis, but its overexpression provides tumor cells 
with an aggressive survival advantage [10]. Hmoxl, like Nrf2 [11], has a dual role in cancer. Hmoxl can stimulate angiogenesis and is prometastatic in some but not all cancers [12]. CRC with Hmoxl expression has a lower rate of lymphatic tumor invasion and better survival than that without [13]. Welldifferentiated CRC seems to have more total but less nuclear Hmoxl expression than moderately/poorly differentiated CRC [14]. The role of Hmoxl and its interaction with the Nrf2 pathway in CRC remains uncertain. This study determined the mRNA levels of Nrf2, Keap1, Bach1, and Hmoxl, both in the tumor and in normal tissues, to investigate their correlations in CRC.

\section{Materials and Methods}

Eighty-four consecutive subjects with a preoperative diagnosis of CRC were recruited after informed consent had been signed. Histopathologic evaluation was performed based on the diagnostic criteria of the World Health Organization [15], and all of the tumors were diagnosed as adenocarcinoma. The staging assessment was carried out according to the American Joint Committee on Cancer TNM-classification (7th edition) [16]. Clinical and pathologic characteristics were reviewed and recorded. Tumor size was defined as the product of the longitudinal and horizontal dimensions. The work was approved by the Institutional Review Board of Chang Gung Memorial Hospital.

2.1. Real-Time Polymerase Chain Reaction (PCR). The tumor and normal colorectal tissue ( $>10 \mathrm{~cm}$ away from the margin of tumor) were embedded in OCT compound (Tissue-Tek, Sakura Finetek USA, Inc., Torrance, CA) within 30 minutes of surgical resection and stored at $-20^{\circ} \mathrm{C}$ for less than 2 weeks before RNA extraction. A $2 \mathrm{~mm}^{3}$ portion of frozen tumor or normal colorectal tissue was minced, and the total RNA was extracted with the RNeasy Mini Kit (Qiagen, Hilden, Germany) and treated with RQ1 RNase-Free DNase (Promega, Madison, WI, USA) at $37^{\circ} \mathrm{C}$ for 10 minutes. The purity of RNA was determined spectrophotometrically. The cDNA was generated by reverse transcription (SuperScript III, Invitrogen, Carlsbad, CA, USA) using $1 \mu \mathrm{g}$ of total RNA. PCR amplification of glyceraldehyde-3-phosphate dehydrogenase (GAPDH) was conducted to confirm the integrity of cDNA. Real-time PCR was performed on a Bio-Rad iQ5 Real-Time PCR Detection System (Bio-Rad Laboratories, Hercules, CA, USA) with the following primers and TaqMan FAM-labeled MGB probes (Life Technologies, Carlsbad, CA, USA): Hs99999903_ml for $\beta$-actin; Hs00202227_ml for Keap1; Hs00975961_g1 for Nrf2; Hs00230917_ml for Bach1; and Hs01110250_ml for Hmoxl. For each $0.5 \mathrm{~mL}$ Eppendorf tube, $12.5 \mu \mathrm{L}$ 2x FastStart Universal Probe Master (Roche), $1.25 \mu \mathrm{L}$ primer and probe mix, $9.25 \mu \mathrm{L}$ RNase-free water, and $2 \mu \mathrm{L}$ of cDNA were added to reach a total volume of $25 \mu \mathrm{L}$. The following cycling conditions were for real-time PCR: preincubation with uracil-N-glycosylase at $50^{\circ} \mathrm{C}$ for 2 minutes and AmpliTaq Gold activation at $95^{\circ} \mathrm{C}$ for 10 minutes followed by 60 cycles of denaturation at $95^{\circ} \mathrm{C}$ for 15 seconds and annealing/extension at $60^{\circ} \mathrm{C}$ for 1 minute. The sizes of amplicons from real-time PCR were checked by gel
TABLE 1: Characteristics of 84 subjects with colorectal cancer.

\begin{tabular}{lc}
\hline Age (years) & $68.3 \pm 13.0$ \\
Gender (male/female) & $48(57 \%) / 36(43 \%)$ \\
CEA & $2.02(0.5-1326.1)$ \\
Clinical stage & \\
$\quad$ I & $11(13 \%)$ \\
II & $26(31 \%)$ \\
III & $33(39 \%)$ \\
IV & $14(17 \%)$ \\
Histological differentiation & \\
Well & $18(21 \%)$ \\
$\quad$ Moderate & $56(67 \%)$ \\
$\quad$ Poorly & $10(12 \%)$ \\
Tumor size, cm & $26.3 \pm 19.7$ \\
\hline
\end{tabular}

electrophoresis for their deviation from those provided by the manufacturer. Duplicated cDNA samples of both the tumor and normal tissues from the same patient and the notemplate control for each gene were included in the same realtime PCR experiment. Baseline and threshold values were automatically determined and the expression level of gene was evaluated through Normalized Gene Expression (ddCT) by the Bio-Rad iQ5 optical system software (version 2.1). The real-time PCR test was duplicated for each sample, and the reported data was the average of two readings (Ct number) adjusted by that of $\beta$-actin.

2.2. Statistical Analysis. Data analyses were performed by SPSS version 18 (SPSS, Inc., Chicago, Illinois, USA) and R Core Team [17]. Chi-square analysis was used to compare discrete variables. The Mann-Whitney and Kruskal-Wallis tests were undertaken to detect the difference between and among variables, respectively. The difference between paired data was detected by Wilcoxon signed ranks test. The correlations between variables were presented by Spearman's correlation coefficients. Logistic regression with stepwise selection [18] by Bayesian information criterion (BIC) was used to determine the significance of the following variables as predictors for distant metastasis in CRC: age, sex, serum CEA level, histological grade, tumor size, and mRNA level of an individual molecule or its ratio to that of $\mathrm{Nrf2}$ expressed as a percentage. $p<0.05$ was considered statistically significant.

\section{Results}

Table 1 shows the characteristics of 84 subjects with CRC. The extracted RNA of all samples had OD260/OD280 values greater than 1.8. Table 2 lists the mRNA levels of molecules. The Bachl mRNA level, when detectable, was higher in the tumor than in normal tissue. The Hmoxl mRNA level was higher in the tumor than in normal tissue whether or not Bach1 mRNA expression was detectable. The Nrf2 mRNA level of normal tissue was higher in the subjects with detectable Bachl mRNA expression than in those without. Table 3 presents the ratios of mRNA levels to Nrf2 levels in percentage. The tumor had higher Keap1/Nrf2 and 
TABLE 2: mRNA levels in the tumor and normal tissues of colorectal cancer with or without detectable Bach1 mRNA.

\begin{tabular}{|c|c|c|c|c|}
\hline & \multirow{2}{*}{\multicolumn{2}{|c|}{$\begin{array}{c}\text { Bach1 mRNA detectable }{ }^{\dagger} \\
\qquad n=62\end{array}$}} & \multirow{2}{*}{\multicolumn{2}{|c|}{$\begin{array}{l}\text { Bachl mRNA not detectable } \\
\qquad n=22\end{array}$}} \\
\hline & & & & \\
\hline & Normal tissue & Tumor & Normal tissue & Tumor \\
\hline Nrf2 & $0.93(0.82-1.03)$ & $0.92(0.75-1.00)$ & $0.95(0.68-1.42)^{* *}$ & $0.90(0.52-1.13)$ \\
\hline Keap1 & $1.00(0.85-1.81)$ & $1.01(0.86-1.08)$ & $0.98(0.83-1.36)$ & $0.99(0.74-1.49)$ \\
\hline Hmoxl & $0.98(0.76-1.12)^{*}$ & $0.92(0.78-1.03)$ & $1.01(0.74-1.48)^{\ddagger}$ & $0.88(0.56-1.10)$ \\
\hline Bach1 & $0.93(0.75-1.04)^{*}$ & $0.94(0.80-1.03)$ & - & - \\
\hline
\end{tabular}

${ }^{\dagger}$ Subjects with Bachl mRNA expression detectable in both the tumor and normal tissues.

${ }^{\ddagger}$ Subjects with Bachl mRNA expression not detectable in the tumor or normal tissue.

${ }^{*}$ Significantly different from the tumor tissue by Wilcoxon signed ranks test.

${ }^{* *}$ Significantly different from subjects with detectable Bachl mRNA expression by Mann-Whitney test.

TABLE 3: Ratios of mRNA levels to that of Nrf2 by percentage in colorectal cancer.

\begin{tabular}{lcccc}
\hline$\%$ & & Keap1/Nrf2 & Bach1/Nrf2 & Hmox1/Nrf2 \\
\hline Normal tissue & & $107.1(88.8-198.9)^{*}$ & $100.0(76.5-107.2)^{*}$ & $105.1(92.7-137.0)^{*}$ \\
Tumor & & $109.6(102.3-146.0)$ & $102.2(95.2-116.0)$ & $100.0(84.4-136.5)$ \\
& Distant metastasis & & & \\
Normal tissue & With & $108.6(101.0-140.2)$ & $100.0(90.0-106.7)$ & $106.3(96.7-137.0)$ \\
& Without & $106.9(88.8-198.9)$ & $100.0(76.5-107.3)$ & $104.3(92.7-135.8)$ \\
Tumor & With & $109.1(105.0-116.0)$ & $102.2(98.0-106.0)$ & $97.4(84.4-111.1)^{* *}$ \\
& Without & $109.6(102.0-146.0)$ & $102.2(95.0-116.0)$ & $101.0(92.7-136.5)$ \\
\hline
\end{tabular}

* Significantly different between the tumor and normal tissues by Wilcoxon signed ranks test.

** Significantly different between tissues of subjects with or without distant metastasis by Mann-Whitney test.

Bach1/Nrf2 but lower Homx1/Nrf2 mRNA ratios compared to the normal tissue. The Hmoxl/Nrf2 mRNA ratio in the tumor was lower in the subjects with distant metastasis than in those without. The mRNA levels of all molecules and the detectability of Bach1 mRNA in both the tumor and normal tissues were not different between subjects with and subjects without distant metastasis. The mRNA levels of all molecules and their ratios to the Nrf2 levels in both the tumor and normal tissues were not different between genders and among histological grades, clinical stages, or smoking statuses.

Figure 1 shows Spearman's correlation coefficients between mRNA ratios. The mRNA levels were all significantly correlated in the tumor and normal tissue. Figure 2 presents the correlations of mRNA levels between the tumor and normal tissues, which were significant in Nrf2, Keap1, and Hmoxl, but not in Bach1. Figure 3 shows the correlations of mRNA levels in the normal and tumor tissues with or without detectable Bach1 mRNA expression. The Nrf2 mRNA level lost significant correlations with those of Keapl and Hmoxl in the tumor tissue of subjects without detectable Bach1 mRNA expression. Figure 4 presents the correlations of mRNA levels in the normal and tumor tissues with and without distant metastasis. In subjects with distant metastasis, the correlations between the mRNA levels of Nrf2 and Hmoxl were not significant in either the tumor or normal tissues.

The logistic regression with stepwise selection by BIC found that the ratio of Hmox1/Nrf 2 mRNA by percentage in the tumor tissue (odds ratio: 0.83 ; 95\% confidence interval: 0.68-0.97) and CEA (1.0027; 1.006-1.064) were predictors for distant metastasis in CRC.

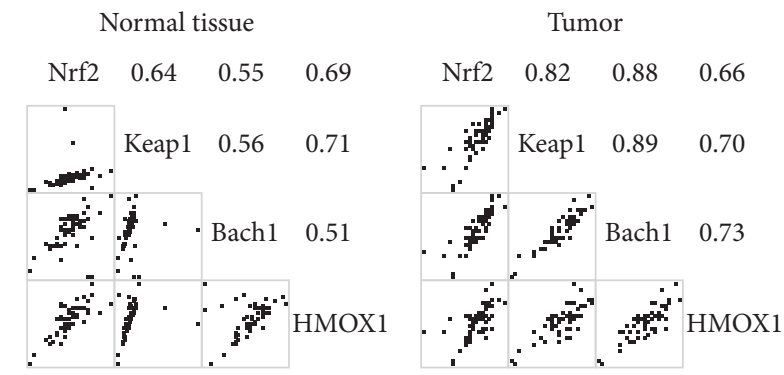

FIGURE 1: Spearman's correlation coefficients of mRNA levels in the tumor and normal tissues. All correlations are significant.

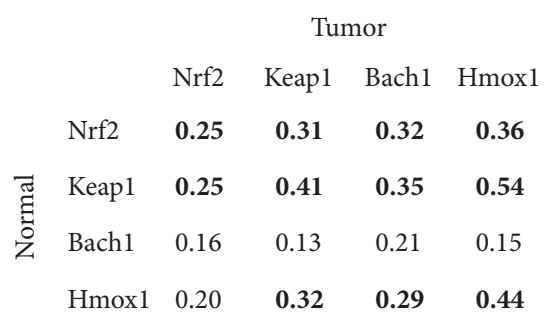

FIGURE 2: Spearman's correlation coefficients of mRNA levels between the tumor and normal tissues. A bold number denotes $p$ value less than 0.05 .

\section{Discussion}

The tumor had lower Hmoxl but higher Bach1 mRNA levels and ratio with Nrf2 than the normal tissue in CRC. In the 


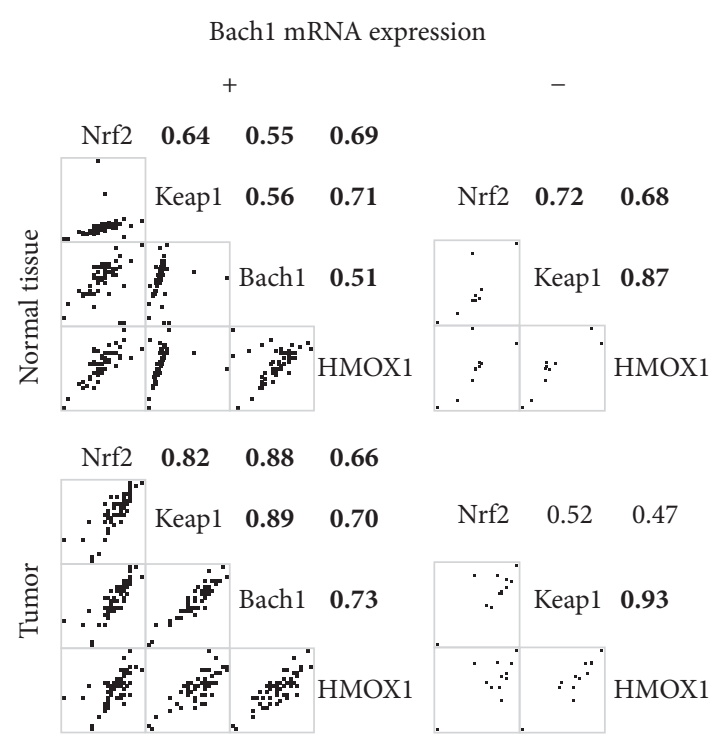

FIGURE 3: Spearman's correlation coefficients of mRNA levels in the tumor and normal tissues of subjects with or without detectable Bachl mRNA expression. A bold number denotes a $p$ value less than 0.05 .

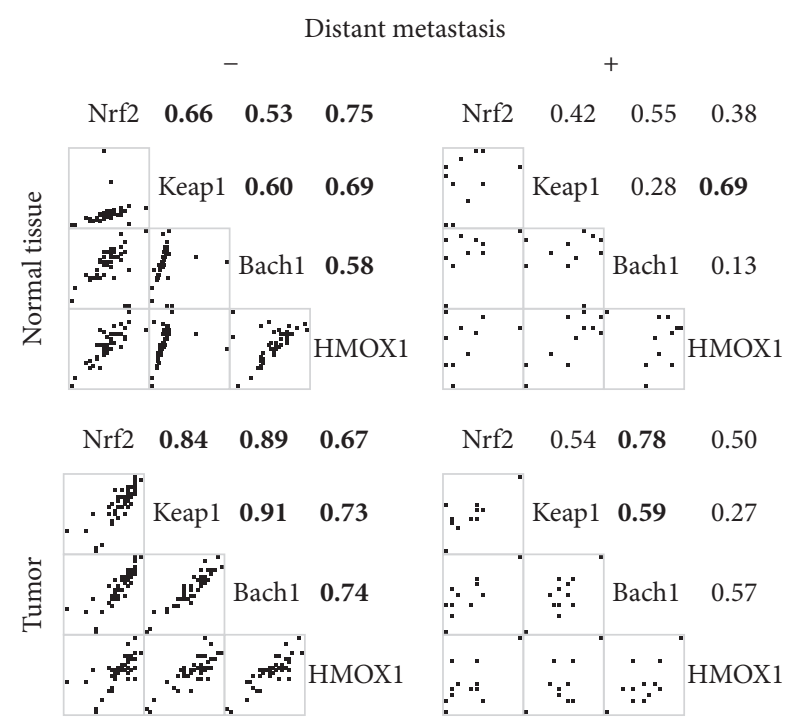

FIGURE 4: Spearman's correlation coefficients of mRNA levels in the tumor and normal tissues of subjects with or without distant metastasis. A bold number denotes a $p$ value less than 0.05 .

absence of detectable Bach1 mRNA expression, the mRNA levels of Keap1, but not Nrf2, retained significant correlation with that of Hmoxl in the tumor tissue of CRC. The significant correlations between the mRNA levels of Hmoxl and Nrf2 were lost in both the tumor and normal tissues of CRC subjects with distant metastasis. The Hmoxl/Nrf2 mRNA ratio in the tumor tissue was lower in CRC subjects with distant metastasis than in those without and was a significant predictor of distant metastasis in CRC.

The development of both sporadic and colitis-associated CRC involves many of the same genetic defects, which may be caused by oxidative stress [2]. Nrf2 is the principal transcription factor affecting the expression of phase II antioxidant enzymes like Hmoxl [19]. Nrf2 can be constitutively overexpressed in cancer cells or tumor tissues and is a protooncogene that can suppress or promote tumor $[10,20]$. Hmoxl can protect tissue against oxidative stress [21] and is a key effector of $\mathrm{Nrf} 2$ upregulation in tumor progression [4]. The Nrf2/Hmoxl axis is a double-edged sword in cancer [4]. Many molecules, such as Keapl, Fyn, and Bach1, whose transcription is regulated by Nrf2, can increase the degradation of Nrf2 or compete with its binding to ARE on the promoter, while other molecules like sequestosome 1 and PALB2 can stabilize Nrf2 [22]. Under oxidative stress, nuclear translocated Hmoxl interacts with Nrf2 and protects it from GSK3 $\beta$-mediated phosphorylation coupled with ubiquitinproteasomal degradation, thereby prolonging its accumulation in the nucleus [8]. The function of Nrf2 as a transcription factor is affected by many proteins and autoregulatory loops [23].

Although epigenetic modification affects the expression of Nrf2 and Keap1 in many ways [24], their mRNA levels are not different between the tumor and normal tissues. The tumor had lower Hmoxl and higher Bachl mRNA levels than the normal tissue. Hmoxl had mRNA expression lower in the tumor than in the normal tissue by gene array [25]. Bach1 plays a critical role in the negative regulation of Hmoxl transcription through the StRE- (stress-responsive element-) Bach1-Nrf2 axis [21]. The Bach1 mRNA level is regulated by many factors like Nrf2, heme, Raf kinase inhibitor protein (RKIP), miR-155-5p, and itself [7, 26-28]. Bach1 mRNA expressions were undetectable in some subjects (22/84) and were insignificantly correlated between the tumor and normal tissues, suggesting that the regulation of Bachl expression is different between tissues and among subjects of CRC. In the tumor tissue with undetectable Bachl mRNA expression, the correlation between the mRNA levels of Keapl and Hmoxl was the only one that remained significant. In keratinocytes, knockdown of Bach1, Keapl, and Nrf2 has fold changes of Hmoxl mRNA transcription of 136.4, 2.3, and 0.4, respectively [29]. Without Bach1 expression, Keap1 may become the major regulator of Hmoxl mRNA transcription in CRC tumor tissue. Bachl may play a role in the transcriptional regulation of Hmoxl by the Nrf2-Keapl pathway in the tumorous CRC tissue, but not the normal tissue.

Hmox-1 may increase the metastatic potential of cancer due to its proangiogenic property [3]. However, Hmox-1 expression in CRC is associated with prolonged survival and a low rate of lymphatic tumor invasion [13]. Inhibition of Hmoxl can increase the liver metastasis of CRC in mice [30]. The role of Hmoxl in tumor metastasis remains unclear [12]. Bach1 promotes the liver metastasis of CRC by upregulating c-Myc and SOX4 [31]. RKIP, a downregulator of Bachl expression [27], is reduced in the metastatic tumor of CRC [32]. High level of miR-155-5p, which can target Bach1 mRNA [28], in the tumor tissue of CRC is associated with increased lymph node metastasis [33]. The role of Bachl in the metastasis of CRC is uncertain. The role of Nrf2 in tumor metastasis remains to be clarified [10]. Frequent hypermethylation of Keapl promoter reduces its mRNA transcription but is not 
associated with clinicopathological features in CRC [34]. The mRNA levels of Hmoxl and molecules of the Nrf2 pathway in either the tumor or normal tissues were not predictors for distant metastasis in CRC.

Hmoxl has many regulatory domains other than StRE on its promoter and its mRNA transcription is affected by many factors like NF- $\kappa$ B, IL-6, and STAT3 [21]. Conversely, the transcription of Nrf2 can be regulated by factors other than itself $[23,24]$ and increased by K-Ras, B-Raf, and Myc in tumor cells [22]. Epigenetic alterations by miRNAs upregulate Bach1 and downregulate Keap1, K-ras, STAT3, and Myc expression in CRC [28]. ROS can regulate the activity of Nrf2 through redox factor-1 [35]. Variable ratios of Hmoxl/Nrf2 mRNA levels may represent that the transcription regulations of Hmoxl and Nrf2 are modified by factors other than Nrf2, Keapl, and Bachl. A disease is a consequence of the complex intracellular network rather than a single gene [36] and so is cancer metastasis [37]. Although the Nrf2/Hmoxl axis is important in tumor progression [4], Nrf2 and Hmoxl have uncertain roles in cancer metastasis [10, 12]. Many factors involving the transcriptional regulation of Nrf2 and Hmoxl play roles in the metastasis of CRC $[10,25,31,38-40]$. The lack of significant correlations between Nrf2 and Hmoxl mRNA levels in both the tumor and normal tissues suggests that the transcriptional regulation of Hmoxl by Nrf2 is loose in CRC subjects with distant metastasis. The ratio of Hmoxl/Nrf2 mRNA levels in the tumor tissue may reflect a difference in their transcriptional regulation and was a negative predictor for distant metastasis in CRC.

ROS plays an important role in the carcinogenesis of CRC [2] and metastasis in cancer [35]. Nrf2 and Hmoxl can protect cells from oxidative stress [4] and play roles in tumor metastasis $[4,10]$. The transcriptional regulations of Nrf2 and Hmoxl are complex and modified by many factors $[21-24,28,35]$. The Hmox1/Nrf2 mRNA ratio in the tumor tissue may be a useful indicator for distant metastasis in CRC. The differential expressions of Nrf2 and Hmoxl mRNA and their relation to distant metastasis in CRC warrant further investigation.

\section{Competing Interests}

The authors declare that they have no competing interests.

\section{Acknowledgments}

This investigation was supported by grants from Chang Gung Memorial Hospital, Keelung (CMRPG2A0251, CMRPG2A0252, and CMRPG2A0253).

\section{References}

[1] A. Bhattacharyya, R. Chattopadhyay, S. Mitra, and S. E. Crowe, "Oxidative stress: an essential factor in the pathogenesis of gastrointestinal mucosal diseases," Physiological Reviews, vol. 94, no. 2, pp. 329-354, 2014.

[2] T. A. Ullman and S. H. Itzkowitz, "Intestinal inflammation and cancer," Gastroenterology, vol. 140, no. 6, pp. 1807-1816, 2011.

[3] H. Was, J. Dulak, and A. Jozkowicz, "Heme oxygenase-1 in tumor biology and therapy," Current Drug Targets, vol. 11, no. 12, pp. 1551-1570, 2010.
[4] A. L. Furfaro, N. Traverso, C. Domenicotti et al., "The Nrf2/HO1 axis in cancer cell growth and chemoresistance," Oxidative Medicine and Cellular Longevity, vol. 2016, Article ID 1958174, 14 pages, 2016.

[5] H. Motohashi and M. Yamamoto, "Nrf2-Keap1 defines a physiologically important stress response mechanism," Trends in Molecular Medicine, vol. 10, no. 11, pp. 549-557, 2004.

[6] K. Itoh, N. Wakabayashi, Y. Katoh et al., "Keap1 represses nuclear activation of antioxidant responsive elements by Nrf2 through binding to the amino-terminal Neh2 domain," Genes \& Development, vol. 13, no. 1, pp. 76-86, 1999.

[7] G. P. Sykiotis and D. Bohmann, "Stress-activated cap'n'collar transcription factors in aging and human disease," Science Signaling, vol. 3, no. 112, p. re3, 2010.

[8] C. Biswas, N. Shah, M. Muthu et al., "Nuclear heme oxygenase1 (HO-1) modulates subcellular distribution and activation of Nrf2, impacting metabolic and anti-oxidant defenses," The Journal of Biological Chemistry, vol. 289, no. 39, pp. 26882-26894, 2014.

[9] L. L. Dunn, R. G. Midwinter, J. Ni, H. A. Hamid, C. R. Parish, and R. Stocker, "New insights into intracellular locations and functions of heme oxygenase-1," Antioxidants and Redox Signaling, vol. 20, no. 11, pp. 1723-1742, 2014.

[10] H.-K. Na and Y.-J. Surh, "Oncogenic potential of Nrf2 and its principal target protein heme oxygenase-1," Free Radical Biology and Medicine, vol. 67, pp. 353-365, 2014.

[11] A. Lau, N. F. Villeneuve, Z. Sun, P. K. Wong, and D. D. Zhang, "Dual roles of Nrf2 in cancer," Pharmacological Research, vol. 58, no. 5-6, pp. 262-270, 2008.

[12] A. Jozkowicz, H. Was, and J. Dulak, "Heme oxygenase-1 in tumors: is it a false friend?" Antioxidants and Redox Signaling, vol. 9, no. 12, pp. 2099-2117, 2007.

[13] J. C. Becker, H. Fukui, Y. Imai et al., "Colonic expression of heme oxygenase-1 is associated with a better long-term survival in patients with colorectal cancer," Scandinavian Journal of Gastroenterology, vol. 42, no. 7, pp. 852-858, 2007.

[14] H. Yin, J. Fang, L. Liao, H. Maeda, and Q. Su, "Upregulation of heme oxygenase- 1 in colorectal cancer patients with increased circulation carbon monoxide levels, potentially affects chemotherapeutic sensitivity," BMC Cancer, vol. 14, no. 1, pp. 1-27, 2014.

[15] F. T. Bosman, F. Carneiro, R. Hruban, and N. Theise, WHO Classification of Tumours of the Digestive System, 2010.

[16] L. L. Gunderson, J. M. Jessup, D. J. Sargent, F. L. Greene, and A. K. Stewart, "Revised TN categorization for colon cancer based on national survival outcomes data," Journal of Clinical Oncology, vol. 28, no. 2, pp. 264-271, 2010.

[17] R Core Team, R: A Language and Environment for Statistical Computing, R Foundation for Statistical Computing, 2014.

[18] V. N. Venables and B. D. Ripley, Modern Applied Statistics with S, Springer, New York, NY, USA, 4th edition, 2002.

[19] T. Rangasamy, C. Y. Cho, R. K. Thimmulappa et al., "Genetic ablation of Nrf2 enhances susceptibility to cigarette smokeinduced emphysema in mice," Journal of Clinical Investigation, vol. 114, no. 9, pp. 1248-1259, 2004.

[20] P. Shelton and A. K. Jaiswal, "The transcription factor NF-E2related factor 2 (nrf2): a protooncogene?" The FASEB Journal, vol. 27, no. 2, pp. 414-423, 2013.

[21] S. W. Ryter, J. Alam, and A. M. K. Choi, "Heme oxygenase1/carbon monoxide: from basic science to therapeutic applications," Physiological Reviews, vol. 86, no. 2, pp. 583-650, 2006. 
[22] S. K. Niture, R. Khatri, and A. K. Jaiswal, "Regulation of Nrf2an update," Free Radical Biology and Medicine, vol. 66, pp. 3644, 2014.

[23] D. Papp, K. Lenti, D. Módos et al., "The NRF2-related interactome and regulome contain multifunctional proteins and finetuned autoregulatory loops," FEBS Letters, vol. 586, no. 13, pp. 1795-1802, 2012.

[24] Y. Guo, S. Yu, C. Zhang, and A.-N. T. Kong, "Epigenetic regulation of Keap1-Nrf2 signaling," Free Radical Biology and Medicine, vol. 88, pp. 337-349, 2015.

[25] D. Horst, J. Budczies, T. Brabletz, and F. Hlubek, "Invasion associated up-regulation of nuclear factor $\kappa \mathrm{B}$ target genes in colorectal cancer," Cancer, vol. 115, no. 21, pp. 4946-4958, 2009.

[26] Y. Zhou, H. Wu, M. Zhao, C. Chang, and Q. Lu, "The bach family of transcription factors: a comprehensive review," Clinical Reviews in Allergy \& Immunology, vol. 50, no. 3, pp. 345-356, 2016.

[27] J. Lee, J. Lee, K. S. Farquhar et al., "Network of mutually repressive metastasis regulators can promote cell heterogeneity and metastatic transitions," Proceedings of the National Academy of Sciences of the United States of America, vol. 111, no. 3, pp. E364-E373, 2014.

[28] Y. Okugawa, W. M. Grady, and A. Goel, "Epigenetic alterations in colorectal cancer: emerging biomarkers," Gastroenterology, vol. 149, no. 5, pp. 1204-1225e12, 2015.

[29] A. K. MacLeod, M. McMahon, S. M. Plummer et al., "Characterization of the cancer chemopreventive NRF2-dependent gene battery in human keratinocytes: demonstration that the KEAP1-NRF2 pathway, and not the BACH1-NRF2 pathway, controls cytoprotection against electrophiles as well as redoxcycling compounds," Carcinogenesis, vol. 30, no. 9, pp. 15711580, 2009.

[30] T. Ishikawa, N. Yoshida, H. Higashihara et al., "Different effects of constitutive nitric oxide synthase and heme oxygenase on pulmonary or liver metastasis of colon cancer in mice," Clinical and Experimental Metastasis, vol. 20, no. 5, pp. 445-450, 2003.

[31] J. Yun, Y. N. Jo, S. Park et al., "Bach1 promotes liver metastasis of colorectal cancer cells by regulating c-Myc and SOX4," Cancer Research, vol. 73, no. 8, supplement, p. 3878, 2014.

[32] F. Al-Mulla, S. Hagan, A. I. Behbehani et al., "Raf kinase inhibitor protein expression in a survival analysis of colorectal cancer patients," Journal of Clinical Oncology, vol. 24, no. 36, pp. 5672-5679, 2006.

[33] H. Shibuya, H. Iinuma, R. Shimada, A. Horiuchi, and T. Watanabe, "Clinicopathological and prognostic value of microRNA-21 and microRNA-155 in colorectal cancer," Oncology, vol. 79, no. 3-4, pp. 313-320, 2011.

[34] N. Hanada, T. Takahata, Q. Zhou et al., "Methylation of the KEAP1 gene promoter region in human colorectal cancer," $B M C$ Cancer, vol. 12, no. 1, article 66, 2012.

[35] P. D. Ray, B.-W. Huang, and Y. Tsuji, "Reactive oxygen species (ROS) homeostasis and redox regulation in cellular signaling," Cellular Signalling, vol. 24, no. 5, pp. 981-990, 2012.

[36] A.-L. Barabási, N. Gulbahce, and J. Loscalzo, "Network medicine: a network-based approach to human disease," Nature Reviews Genetics, vol. 12, no. 1, pp. 56-68, 2011.

[37] D. Spano, C. Heck, P. De Antonellis, G. Christofori, and M. Zollo, "Molecular networks that regulate cancer metastasis," Seminars in Cancer Biology, vol. 22, no. 3, pp. 234-249, 2012.

[38] Y. Fan, R. Mao, and J. Yang, "NF- $\kappa$ B and STAT3 signaling pathways collaboratively link inflammation to cancer," Protein and Cell, vol. 4, no. 3, pp. 176-185, 2013.
[39] M. Rokavec, M. G. Öner, H. Li et al., "IL-6R/STAT3/miR34a feedback loop promotes EMT-mediated colorectal cancer invasion and metastasis," The Journal of Clinical Investigation, vol. 124, no. 4, pp. 1853-1867, 2014.

[40] J. Y. Fang and B. C. Richardson, "The MAPK signalling pathways and colorectal cancer," The Lancet Oncology, vol. 6, no. 5, pp. 322-327, 2005. 


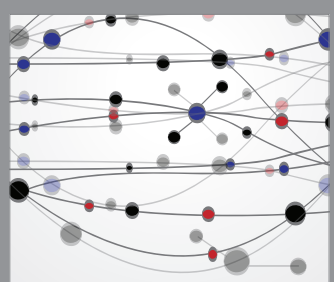

The Scientific World Journal
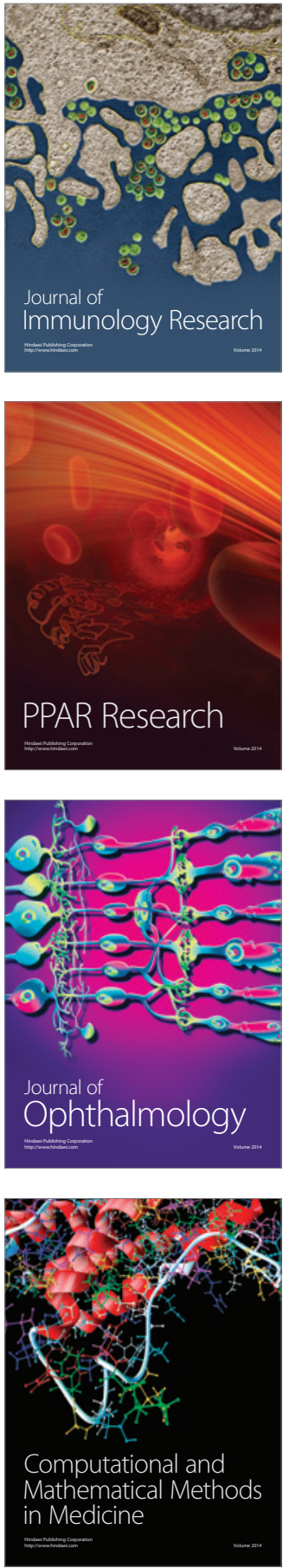

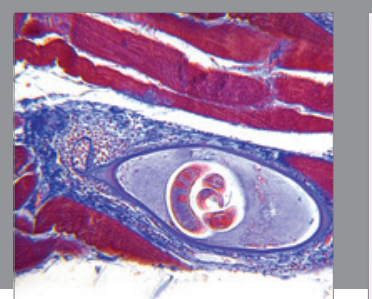

Gastroenterology Research and Practice

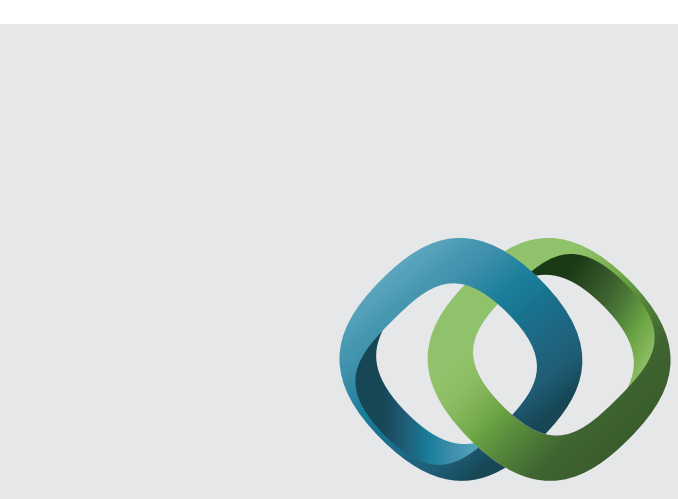

\section{Hindawi}

Submit your manuscripts at

http://www.hindawi.com
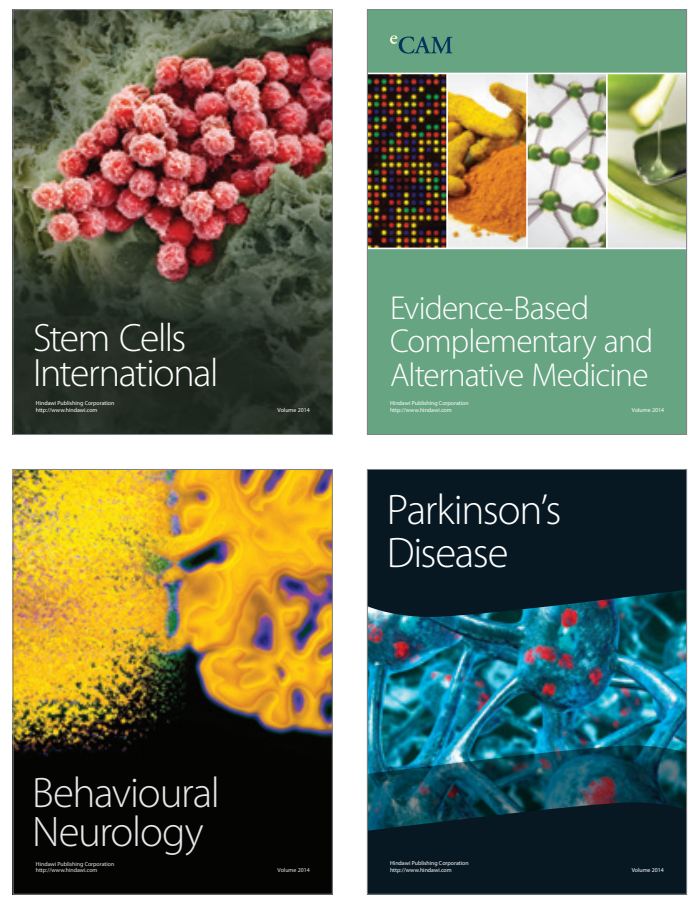
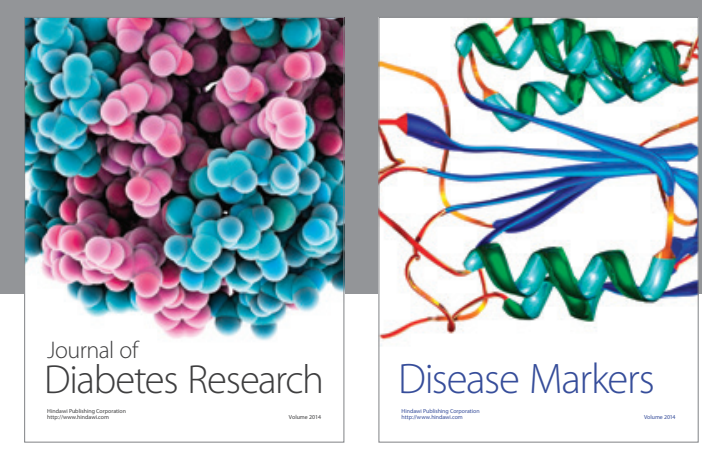

Disease Markers
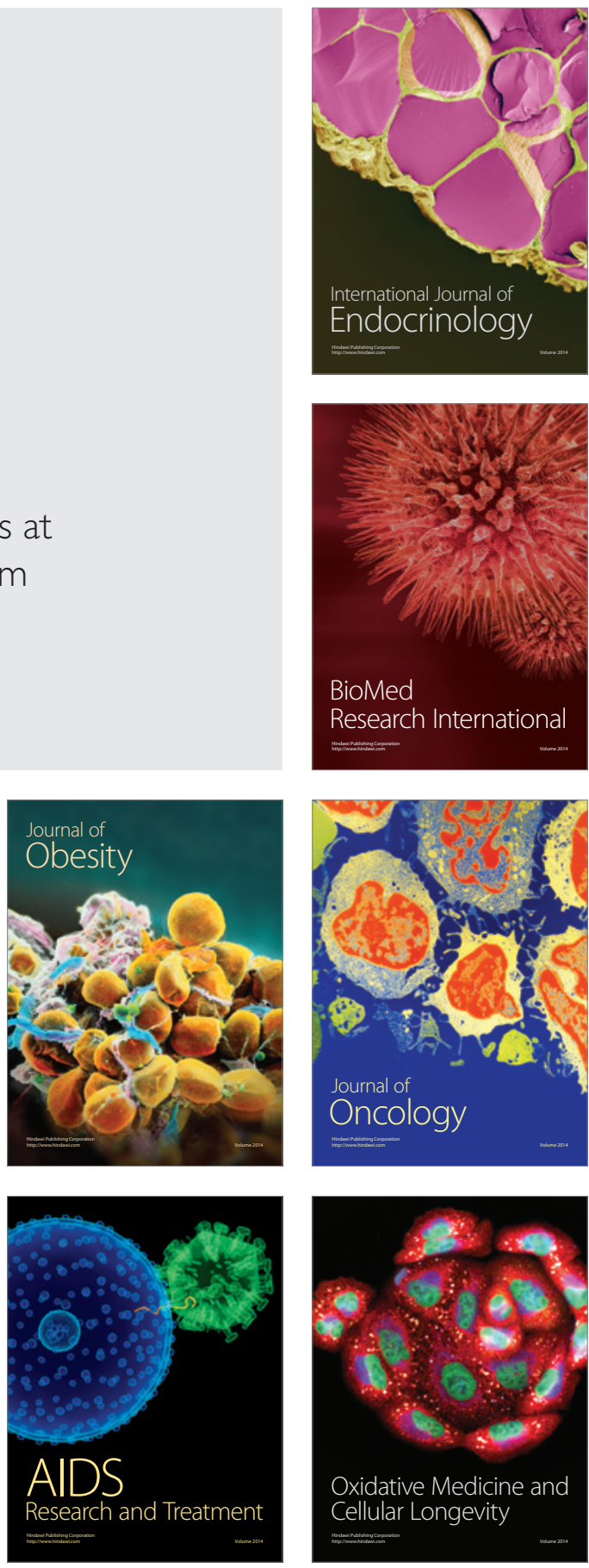